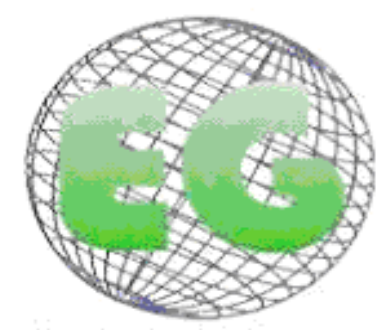

ISSN 1695-6141

$\mathrm{N}^{\circ} 24$
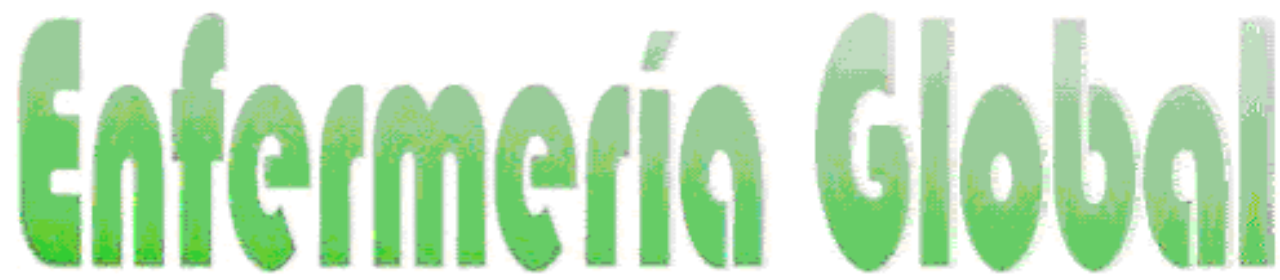

Revista electrónica trimestral de Enfermería

Octubre 2011

www.um.es/egloball

\title{
Evaluación funcional de sistemas sensoriales mediante potenciales evocados en niños con trastornos del espectro autista
}

Functional assessment of sensorial systems through evoked potentials in children

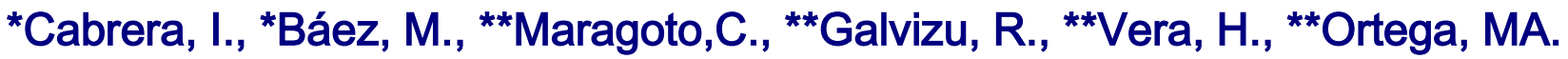 \\ *Servicio de Neurofisiología Clínica. ${ }^{* *}$ Clínica de Neuropediatría. Centro Internacional de Restauración \\ Neurológica. (CIREN). Ciudad de La Habana, Cuba.
}

\begin{abstract}
Palabras clave: Trastorno del espectro autista; potencial evocado auditivo; sistema soméstesico. Keywords: Autistic spectrum disorders; somatosensory evoked potentials; somesthetic system
\end{abstract}

\section{RESUMEN}

Objetivo: Evaluar mediante el empleo de la técnica de registro de potencial evocado somatosensorial de nervio tibial posterior (PESSt) la posible afectación sensorial del sistema somestésico en niños con trastornos del lenguaje, en particular con trastornos del espectro autista (TEA).

Sujetos y Métodos: Realizamos un estudio retrospectivo longitudinal en 40 niños remitidos al Laboratorio de Potenciales Evocados del CIREN en el período comprendido entre agosto del 2000 y mayo del 2009 por presentar trastornos en la adquisición del lenguaje, en su mayoría dentro del espectro autista. La muestra estuvo conformada por 24 varones y 16 hembras (edades entre 2 y 13 años). Para la obtención del PESSt se colocaron electrodos de registro en región lumbar L4- T12 (derivación de plexo) y en región cortical Cz- Fz, estimulando el nervio tibial posterior. Adicionalmente se realizó el potencial evocado auditivo de tallo cerebral (PEATC) para descartar la presencia de afectaciones en la vía auditiva.

Resultados: Del total de niños estudiados el $92.5 \%$ mostró alteraciones en el PESSt, de las cuales el $65 \%$ estuvo relacionada con el incremento del tiempo de conducción central (TCC), un $10 \%$ tuvo ausencia de respuesta cortical bilateral, y el $12,5 \%$ presentó distorsión morfológica de la respuesta evocada cortical.

En tanto el PEATC se realizó a 29 de los 40 niños estudiados, observando que el 75,8 \% fueron normales y solo el 24,1\% presentaron anomalías en el estudio, de estos 5 estuvieron relacionados con afectación de la onda I, otro presentaba hipoacusia bilateral y el caso restante no tuvo respuesta evocada, predominando la afectación de forma bilateral.

Conclusiones: Nuestros resultados muestran una alta incidencia de afectación en el sistema somestésico de niños con TEA. La afectación de este sistema fue mayor que la de los auditivos en los pacientes estudiados.

\section{ABSTRACT}

Objective: To evaluate the possible sensorial affectation of the somesthetic system in children with speech disorders, mainly with the autistic spectrum disorders (ASD)through the use of the recording technique for somatosensory evoked potential (SEP). 
Subjects and methods: We performed a longitudinal retrospective study on 40 children remitted to CIREN'S Laboratory of Evoked Potentials from August 2000 to May 2009. The children presented disorders in language acquisition, most of them within the ASD. The sample was composed of 24 males and 16 females (aged between 2 and 13 years of age). To obtain SEP, recording electrodes were applied on the L4-T12 lumbar region (plexus derivation) and in $\mathrm{Cz}-\mathrm{Fz}$ cortical region, by stimulating the posterior tibial nerve. Additionally, the auditory brainstem response (ABR) was performed to discard functional disorders of the auditory pathway.

Results: $92 \%$ of the children studied showed alterations in SEP, of which $65 \%$ was related to an increment of central conduction time (CCT); $10 \%$ had no bilateral cortical response and $12.5 \%$ presented morphologic distortion of the evoked cortical response.

ABR was conducted on 29 of the 40 studied children, and it was observed that $75.8 \%$ were normal and only $24.1 \%$ presented anomalies. Of these, 5 were related to affectation of wave I, another presented bilateral hypoacusia and the remaining one had no evoked response, where the bilateral form of dysfunction predominated.

Conclusions: Our results suggest a possible relation between the dysfunction of the somesthetic system and language retard. The affectation of this system was greater than the auditory ones in the patients studied.

\section{INTRODUCCIÓN}

Uno de los rasgos característicos del ser humano y que nos diferencia de los animales es la capacidad de comunicarnos a través del lenguaje hablado. El lenguaje se adquiere fundamentalmente en los primeros años de vida ${ }^{1}$.

Para que el lenguaje se desarrolle en forma normal es necesario una audición adecuada desde el nacimiento, un sistema nervioso intacto, control de estructuras físicas y fisiológicas requeridas para un habla intelegible y estímulo ambiental adecuado ${ }^{1}$

La audición juega un papel crítico en el desarrollo del lenguaje y los efectos de una hipoacusia neurosensorial permanente sobre el desarrollo del lenguaje pueden ser considerables ${ }^{1}$.

El autismo es un patrón de retraso del desarrollo de aspecto biológico y etiológico diverso. El trastorno generalmente está caracterizado por un comienzo temprano de déficit cualitativo en la interacción social y conductas estereotipadas, pero que también pueden manifestarse en el sistema inmune, gastrointestinal, y de las funciones sensoriales ${ }^{2,3}$, Autismo, Asperger (AS) y Trastornos Profundos del Desarrollo (TPD) que constituyen los Trastornos del Espectro Autista ${ }^{4}$.

Numerosas especulaciones existen en relación al origen del déficit en el procesamiento sensorial que experimentan los individuos con diagnóstico de autismo y trastornos relacionados. Estas especulaciones están basadas en diferentes hipótesis de disfunción como factor causal e incluyen problemas de modulación de los estímulos sensoriales, déficit en los procesos de discriminación sensorial, elevados niveles de alerta, atención selectiva, déficit en los procesos de entrada, sensori-motriz, y desequilibrio en los procesos de excitación/inhibición en sistemas neurales claves, entre otros. Existen pocas referencias en la literatura del compromiso del sistema somestésico en estos niños ${ }^{5}$

Proponemos evaluar mediante el registro de Potenciales Evocados Auditivos de Tallo Cerebral (PEATC) y Potenciales Evocados Somatosensoriales de nervio tibial posterior (PESSt) las alteraciones antes descritas en el procesamiento sensorial.

\section{MATERIALES Y MÉTODOS}

Realizamos un estudio retrospectivo longitudinal en 40 niños remitidos al Laboratorio de Potenciales Evocados del CIREN en el período comprendido entre marzo del 2000 y mayo 
del 2009 por presentar trastornos del espectro autista. Las características de la muestra aparecen resumidas en la tabla $\mathrm{I}$.

Tabla I. Características de la muestra estudiada

\begin{tabular}{|l|l|l|}
\hline \multicolumn{2}{|l|}{ SEXO } & $\begin{array}{l}\text { RANGO DE } \\
\text { EDAD } \\
\text { (años) }\end{array}$ \\
\hline \hline M & F & $2-13$ \\
\hline 24 & 16 & \\
\hline
\end{tabular}

A los mismos se les realizó el PEATC y PESSt siguiendo la rutina establecida en el manual de procedimientos del laboratorio.

Los registros del PEATC se realizaron con los sujetos acostados en posición decúbito supino, con electrodos de registro en el lóbulo de las orejas, referidos a la posición Cz, y Fpz como tierra (según el sistema internacional 10-20 para colocación de electrodos), y se empleó el sistema para medición de potenciales evocados Neuropack M1 (Nihon Kohden, Japan). Se aplicaron clicks alternos monoauricularmente, promediándose un total de 2000 respuestas divididas en 2 corridas, lo que permitió corroborar la replicabilidad de sus componentes.

Para evaluar el PESSt se colocaron electrodos en región lumbar L4-T12 y en región cortical $\mathrm{Cz}$, referida a Fz con estimulación del nervio tibial posterior por detrás del maleolo medial.

Las condiciones de registro aparecen resumidas en la tabla II.

Tabla II. Condiciones de registro del PEATC y el PESSt

\begin{tabular}{|l|l|l|}
\hline & PEATC & PESSt \\
\hline $\begin{array}{l}\text { Tiempo de análisis } \\
\text { (ms) }\end{array}$ & 10 & 200 \\
\hline $\begin{array}{l}\text { No. de } \\
\text { Promediaciones }\end{array}$ & 1000 & 1000 \\
\hline $\begin{array}{l}\text { Estímulo } \\
\text { Frecuencia del } \\
\text { estímulo }(\mathrm{Hz})\end{array}$ & Clicks alternos & Pulso eléctrico \\
\hline $\begin{array}{l}\text { Duración del } \\
\text { estímulo }(\mathrm{ms})\end{array}$ & 0.1 & 2 \\
\hline Sensibilidad $(\mu \mathrm{V} / \mathrm{div})$ & 10 & 0.2 \\
\hline $\begin{array}{l}\text { Filtros pasabanda } \\
\text { (Hz) }\end{array}$ & $100-3000$ & $1-500$ \\
\hline
\end{tabular}

En el caso del PEATC los parámetros evaluados fueron latencia absoluta y amplitud picolínea de base de los componentes fundamentales registrados (ondas I, III y V), así como duración de los intervalos interpicos I-III, III-V y I-V. 
Para el PESSt se evaluó la latencia del componente lumbar (N20) y cortical (P40) así como el tiempo de conducción central (TCC).

\section{RESULTADOS Y DISCUSIÓN}

Encontramos que en el PEATC el estudio fue negativo en el $75,8 \%$ y solo el $24,1 \%$ presentaron anomalías en el estudio. De estos, 5 estuvieron relacionados con afectación de la onda I, otro presentaba hipoacusia bilateral y el caso restante no tuvo respuesta evocada, predominando la afectación de forma bilateral (tabla III).

Tabla III. Resultados del PEATC. Distribución porcentual.

\begin{tabular}{|l|l|l|}
\hline PEATC & No de casos & $\%$ \\
\hline Negativo & 22 & 75,8 \\
\hline $\begin{array}{l}\text { Afectación de la } \\
\text { Onda I }\end{array}$ & 5 & 17,2 \\
\hline Ausencia de respuesta & 2 & 6,8 \\
\hline
\end{tabular}

En tanto del total de niños estudiados, el $92.5 \%$ mostró alteraciones en el PESSt, de las cuales el $65 \%$ estuvo relacionado con el incremento del (TCC), un $10 \%$ tuvo ausencia de respuesta cortical bilateral, y el 12,5 \% presentó distorsión morfológica de la respuesta evocada cortical (tabla IV).

Tabla IV. Resultados del PESSt. Distribución porcentual.

\begin{tabular}{|l|l|l|}
\hline PESSt & No de casos & $\%$ \\
\hline Negativos & 3 & 7,5 \\
\hline Incremento en elTCC & 26 & 65 \\
\hline $\begin{array}{l}\text { Ausencia de respuesta } \\
\text { Cortical bilateral }\end{array}$ & 4 & 10 \\
\hline Distorsión morfológica & 5 & 12,5 \\
\hline
\end{tabular}

Nuestros resultados sugieren que en el mayor porcentaje de casos estudiados no se detectaron alteraciones de la vía auditiva a nivel de tallo encefálico, lo cual contradice algunos reportes de la literatura.

Sin embargo, sí se aprecia una mayor incidencia de alteraciones en el PESSt en presencia de un trastorno del espectro autista. Estos resultados se encuentran reflejados en los gráficos I y II. 
Gráfico I. Número de sujetos con afectación del PEATC y el PESSt

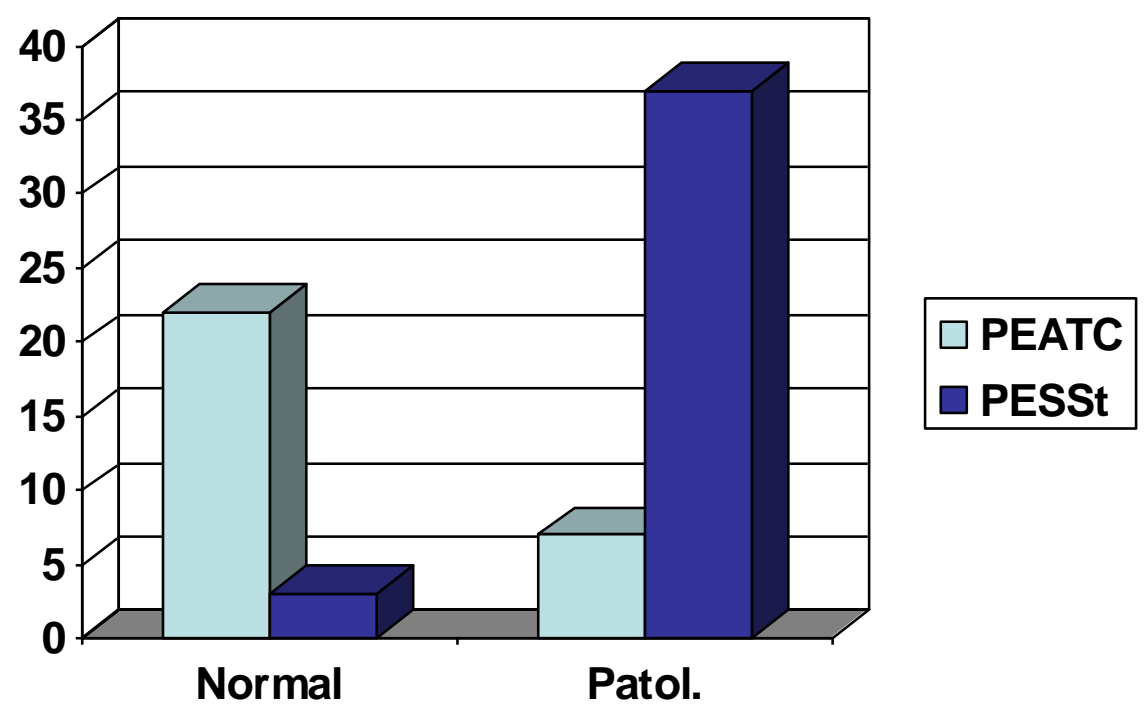

Grafico II. Porciento de afectación del PEATC y el PESSt

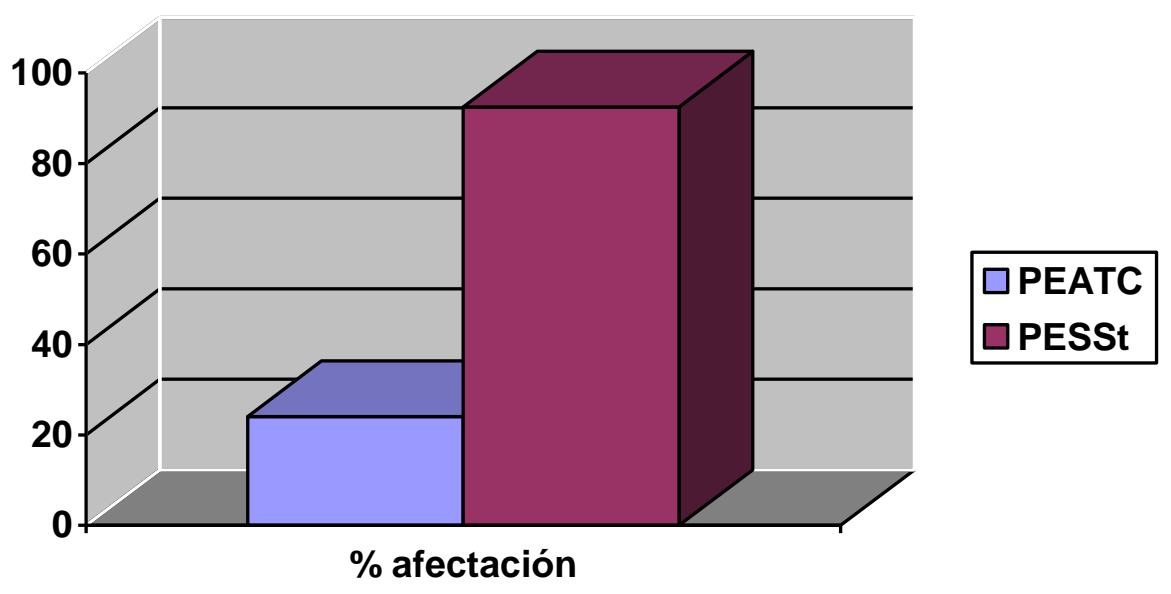

Kern et al. (2006) reportan déficit en los sistemas auditivo, táctil y en el procesamiento oral, que difieren significativamente en comparación con sujetos de control ${ }^{4}$.

Tomchek \& Dunn (2007) estudiaron a 281 niños con autismo y sus controles y encontraron que $92 \%$ de los ítems de la versión breve del Perfil Sensorial (Dunn, 1999) diferenciaba entre niños con y sin autismo y las diferencias más grandes estaban en las secciones de baja respuesta/busca sensaciones, bajo filtro auditivo y sensitividad táctil ${ }^{6}$.

Otro estudios reportan déficit sensoriales principalmente en el sistema táctil, olfativo, gestatorio y visual sugiriendo un déficit que incluye muchas modalidades y que esta presente a lo largo del desarrollo ${ }^{6}$ 
En el sistema somatosensorial, Miyazaki y cols. estudiaron potenciales evocados somatosensoriales por medio de estimulación del nervio mediano. Estos autores reportaron que casi la mitad de los niños con autismo estudiados presentaron anormalidades asociadas a hiperactividad hemisférica derecha, en comparación con la izquierda, especialmente en el área somatosensorial primaria ${ }^{7}$.

Nuestro trabajo está en consonancia con el de estos autores pues se demuestra que existe una afectación en la vía somestésica, en este caso relacionada con la exploración del potencial evocado somatosensorial estimulando el nervio tibial posterior, lo que de alguna manera refleja la disfunción sensorial que presentan estos pacientes.

\section{CONCLUSIONES}

El potencial evocado somato sensorial evidenció anomalías electrofisiológicas que corroboraron el compromiso funcional predominante de esta vía en niños con TEA en relación con las afectaciones del sistema auditivo.

\section{REFERENCIAS BIBLIOGRÁFÍCAS}

1. Carbona, J. El lenguaje del niño y sus trastornos, Texto en prensa para la 3ra edición de "Neurología Pediátrica", N. Fejerman y E. Fernández-Alvarez, Ed. Panamericana 2006.

2. Greenspan, S. \& Wieder, S. (2006). Engaging autism: Using the Floortime approach to help children relate, communicate, and think. Cambridge, MA: Perseus Books.

3. Herbert, M. (2005). Autism, a brain disorder or a disorder that affects the brain? Clinical Neuropsychiatry, 2, 354-369.

4. Kern, J. K. Trivedi, H. Garver, C. R. Grannemann, B. D. Andrews, A. A. Savla, J. S. Johnson, G.S. Jyutika, A. Mehta, J. A. Schroeder, J. L. (2006). The pattern of sensory processing abnormalities in autism, 10, 480. M.

5. Revista chilena de terapia ocupacional [№ 7, noviembre 2007] Escuela de Terapia ocupacional / Facultad de Medicina - Universidad de Chile. Déficit de procesamiento sensorial en el espectro del autismo Consideraciones Etiológicas relacionadas a la disfunción sensorial en individuos con autismo

6. Tomchek, S. \& Dunn, W. (2007). Sensory Processing in Children With and Without Autism: A comparative study using the short sensory profile. American Journal of occupational therapy, 61 (2).

7. Miyazaki M, Fujii E, Saijo T, Mori K, Hashimoto T, Kagami S, Kuroda Y. Shortlatency somatosensory evoked potentials in infantile autism: evidence of hyperactivity in the right primary somatosensory area. Dev Med Child Neurol. 2007 Jan;49(1):13-7. 
ISSN 1695-6141

๑ COPYRIGHT Servicio de Publicaciones - Universidad de Murcia 